For each image set, the following distances were calculated: (1) fiducial to fiducial, (2) $\mathrm{COM}_{\mathrm{Fids}}$ to each individual fiducial, and (3) $\mathrm{COM}_{\text {Fids }}$ to the prostate COM. The magnitudes of these distances in $\mathrm{CT}_{0}$ were compared to those in $\mathrm{CT}_{\text {Sim }}$ to evaluate migration.

Results: Comparison of 45 inter-seed measurements ( 3 for each patient) from $\mathrm{CT}_{0}$ with those from $\mathrm{CT}_{\mathrm{Sim}}$ demonstrated the absolute value of inter-seed migration averaged $0.9 \mathrm{~mm}$ with a standard deviation (SD) of $0.8 \mathrm{~mm}$. The average accounting for both increases and decreases in inter-seed distances between $\mathrm{CT}_{0}$ and $\mathrm{CT}_{\text {Sim }}$ was near zero $(0.1 \mathrm{~mm}$ with a SD of $1.3 \mathrm{~mm})$, indicating no significant systematic increase or decrease during this period. Similarly, the absolute value of the change in distance between the $\mathrm{COM}_{\mathrm{Fids}}$ and each individual seed averaged $1.2 \mathrm{~mm}$ with a SD of $0.9 \mathrm{~mm}$, and the average of increases and decreases in this distance was $0.0 \mathrm{~mm}$ with SD of $1.5 \mathrm{~mm} .95 \%$ of measured changes in the magnitude of inter-seed and $\mathrm{COM}_{\text {Fids }}$-to-seed distances were less than $3 \mathrm{~mm}$, and $90 \%$ were less than $2 \mathrm{~mm}$. The absolute value of the change in distance between the $\mathrm{COM}_{\text {Fids }}$ and the prostate COM was on average $2.6 \mathrm{~mm}$ with a SD of $2.1 \mathrm{~mm}$. This variation is reasonable considering the uncertainty in prostate localization.

Conclusions: Inter-seed distances changed by less than $1 \mathrm{~mm}$ on average in the first week after implantation. The consistency of the distance between the $\mathrm{COM}_{\mathrm{Fids}}$ and the prostate $\mathrm{COM}$ demonstrates the stability of the seeds within the prostate. CT simulation for treatment planning may occur on the day of fiducial implantation without concern for significant migration of the seeds.

Author Disclosure: A. Havnen, None; S. Liauw, None; C. Pelizzari, None; K. Yenice, None.

\title{
2886 Analysis of Dose Volume Histogram Differences between High Dose Rate Brachytherapy and Intensity Modulated Radiotherapy for Prostate Treatment
}

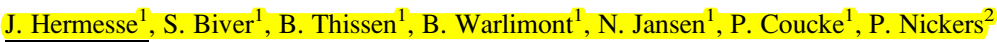

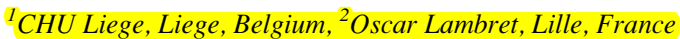

Purpose/Objective(s): We aimed at comparing the dose distribution of HDR brachytherapy and IMRT in prostate cancer.

Materials/Methods: The tomodensitometric data of ten successive patients treated with HDR brachytherapy for prostate cancer were recovered for the dosimetric intercomparison. The Nomos-Corvus treatment planning system (TPS) was used for IMRT planning while dose distribution for HDR brachytherapy was calculated with the Brachyvision TPS. A theoretical dose of 10 Gy applied by 2 Gy per fraction (EQD2) was prescribed on the PTV in the IMRT approach. We selected an $\alpha / \beta$ ratio of 1.5 Gy to calculate a biological equivalent unique dose for HDR brachytherapy ( $5.22 \mathrm{~Gy}$ ). The dose was normalized in order to allow $95 \%$ coverage of the PTV. The dose-volume histograms were calculated for PTV and organs at risk (OARs). For these, doses were converted to EQD2, considering an $\alpha / \beta$ ratio of $3 \mathrm{~Gy}$. Differences between the obtained dose-distributions were compared with a two-sided Student's $t$ test.

Results: Dose heterogeneity is more pronounced with HDR brachytherapy with a mean dose to the PTV of 23.8 Gy as compared to 10.5 Gy with IMRT. Cold spots are similar with both methods: $7.93 \mathrm{~Gy}$ and $8.17 \mathrm{~Gy}$, respectively, with IMRT and HDR brachytherapy $(p=0.6)$. The rectal dose is reduced by HDR brachytherapy as $0.5 \mathrm{cc}$ of the rectum received $5.9 \mathrm{~Gy}$ as compared to $10.2 \mathrm{~Gy}$ with IMRT $(p<0.0001) .8 .08$ Gy was delivered to $20 \%$ of the rectal volume with IMRT as compared to 2.74 Gy with HDR brachytherapy $(p<0.0001)$. The same observation favoring HDR brachytherapy is made for the bladder: $20 \%$ of the organ received 7.11 Gy with IMRT but 1.76 Gy with HDR brachytherapy $(p<0.0001)$. The maximal dose delivered to the urethra is increased by HDR brachytherapy to $13.4 \mathrm{~Gy}$ as compared to $11.1 \mathrm{~Gy}$ with IMRT but mean dose and minimal dose on the urethra are significantly lower with HDR brachytherapy $(p<0.0025)$. The volume of normal tissues receiving 1 Gy was reduced by a factor 8 with HDR brachytherapy $(p<0.0001)$.

Conclusions: HDR brachytherapy allows high hyperdosage sleeves inside the PTV while sparing dramatically the OARs as compared to IMRT. The lower number of expected secondary cancers will thus favor brachytherapy use in exclusive or boosting radiation programs, particularly for younger patients.

Author Disclosure: J. Hermesse, None; S. Biver, None; B. Thissen, None; B. Warlimont, None; N. Jansen, None; P. Coucke, None; P. Nickers, None.

\section{Fractional Integral Target Dose Comparison for I-125, Pd-103 and Cs-131 in Prostate Seed Implants}

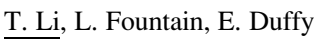

Tuomey Healthcare System, Sumter, SC

Purpose/Objective(s): To use fractional integral target dose to obtain the fraction of energy absorbed by the prostate from integral dose, which is an indication of "radiation delivery efficiency" in brachytherapy. Also to introduce a parameter that shows the fraction of excess energy absorbed in the prostate. Those parameters can be used to aid the clinical choice of isotopes.

Materials/Methods: A total of 15 boost therapy implant cases ( 5 for each isotope) are utilized for this comparison. The TRUS images are obtained intra-operatively using B\&K Ultrasound unit in $5 \mathrm{~mm}$ steps. The planning parameters are defined according to RTOG 0232 guidelines. The prescription doses are: 85 Gy for Cs-131, 108 Gy for I-125 and 100 Gy for Pd-103. The seed strengths employed are: $1.8 \mathrm{U}$ (Cs-131), $1.42 \mathrm{U}$ (Pd-103) and 0.279-0.356 U (I-125). The prostate volume ranges from 25 to $50 \mathrm{cc}$. Planning goals are: $\mathrm{V}_{100} \sim 95 \%, \mathrm{D}_{90} \sim 100 \%$, and prostatic urethra $\mathrm{D}_{10} \sim 150 \%$. Dose calculations are performed using Variseed (ver7.1, VMS, Palo Alto, CA) planning system using AAPM TG-43 formalism. All plans are evaluated to insure the planning goals are met. Uniformity $\left(\mathrm{V}_{200}\right)$, $\mathrm{D}_{90}$ for urethra, and $\mathrm{D}_{30}$ for rectum are also evaluated. Integral target dose are obtained by multiplying the prostate volume $\mathrm{V}$ with mean dose $\mathrm{D}_{\text {mean }}$. Total integral dose is obtained by adopting the point source model from AAPM TG43. The integral dose $\mathrm{E}$ delivered by a single source with unit activity is the integration of $4 \mathrm{Pi} \mathrm{r}^{2} \mathrm{D}(\mathrm{r}) / \mathrm{r}^{2} \mathrm{dr}$ times $1.44 \mathrm{~T}_{1 / 2}$. Let $\mathrm{A}$ be the total activity implanted, the total integral dose will be AE. The fractional integral target dose the prostate receives is defined as $D_{\text {mean }} \mathrm{V} / \mathrm{AE}$. We also define a parameter $\left(\mathrm{D}_{\text {mean }}-\mathrm{mPD}\right) \mathrm{V} / \mathrm{AE}$ that indicates the fraction of excess energy absorbed in the prostate. This parameter shows the amount of energy that is more than needed to achieve the minimum peripheral dose $(\mathrm{mPD})$. 